Instituto Internacional de Investigación y Desarrollo Tecnológico Educativo INDTEC, C.A.

DOI: https://doi.org/10.29394/Scientific.issn.2542-2987.2018.3.7.4.78-98

OAI-PMH: http://www.indteca.com/ojs/index.php/Revista Scientific/oai

\title{
La Música, Herramienta para el Fortalecimiento de Valores en la Educación Básica
}

\author{
Autores: Carol del Carmen Terán González \\ Universidad Nacional Experimental "Rafael María Baralt”, UNERMB \\ carolteranula@gmail.com \\ Trujillo, Venezuela \\ Lom Hill Parra \\ Universidad de los Andes Núcleo Universitario Rafael Rangel, NURR \\ lom 203@hotmail.com \\ Trujillo, Venezuela \\ Josefa María Ramírez Becerra \\ Universidad Nacional Experimental "Rafael María Baralt", UNERMB \\ josefa chepa@hotmail.com \\ Trujillo, Venezuela
}

\section{Resumen}

El propósito del estudio consistió en diseñar alternativas de enseñanzaaprendizaje utilizando la música urbana como herramienta para el fortalecimiento de valores en los estudiantes del 3er grado de la Escuela Bolivariana "Vivienda Rural" estado Trujillo. El estudio se basó en una investigación descriptiva un diseño de campo no experimental. Las unidades de análisis la constituyeron seis (06) docentes que imparten clases en tercer grado. En los resultados obtenidos se logró evidenciar que los docentes del 3er grado hacen uso de distintas estrategias en el aula de clase, lo cual es favorable en el aprendizaje de los niños; sin embargo, la música no es tomada en cuenta como una herramienta de enseñanza en el proceso educativo. Es por ello que, se realizó una propuesta por parte del investigador diseñando actividades tendientes a mejorar la enseñanza-aprendizaje de valores, así como motivar a los estudiantes a realizar sus propias creaciones musicales partiendo de las vivencias diarias.

Palabras clave: música; fortalecimiento de valores; enseñanza aprendizaje; educación. 


\title{
The Music, Tool for Strengthening Value in the Basic Education
}

\begin{abstract}
The intention of the study consisted of designing alternatives of education learning using the urban music as tool for the strengthening value in the students of 3er degree of the School Bolivariana "Rural Housing" been Trujillo. The study based on a descriptive investigation a design of not experimental field. The units of analysis she was constituted by six (06) teachers who give classes in the third degree. In the obtained results it achieved to demonstrate that the teachers of 3er degree use different strategies in the classroom of class, which is favorable in the learning of the children; nevertheless, the music is not taken in account as a tool of education in the educational process. It is for it that, an offer was realized on the part of the investigator designing activities tending to improve the education - learning value, as well as to motivate the students to realizing his own musical creations departing from the daily experiences.
\end{abstract}

Keywords: music; strengthening of values; education learning; education. 


\section{Introducción}

La expresión musical es un espacio escasamente explorado por los docentes de educación básica; de allí, nace esta iniciativa de parte del investigador para brindar ideas acerca de cómo trabajar con la música en el quehacer diario del aula de una manera lúdica y entretenida tanto para los estudiantes como para los docentes. En este momento, la música es una herramienta comunicativa de representación masiva y gran valor, ya que puede congregar a personas de distintas edades, condiciones sociales y provenientes de diferentes partes del mundo.

Por tal razón, se puede reflexionar sobre la música como un lenguaje universal que puede transmitir palabras, también transfiere sentimientos y emociones. Es importante señalar, además, que la poca utilización de la música como recurso educativo en Venezuela, se deba a que el docente de educación básica no posee una formación regular en cuanto a didáctica de la música; esto no debe presumir ningún obstáculo para desarrollar la expresión musical en el aula con los niños, ya que la idea no es formar a los niños como artistas en esas batallas, sino aproximarlos al mundo de la música y utilizar ese lenguaje como una forma de enseñar.

Por otra parte, los juegos musicales para fomentar los valores de los niños en educación básica no requieren de una formación musical por parte del docente. Habitualmente el espacio de mayor recurrencia para ocuparse de la música en el aula, cuando se recurre a ella, es la canción, sobre todo las infantiles; con esto, se pueden incitar actividades que ayuden desde las inteligencias múltiples a internalizar los elementos axiológicos en la formación de los estudiantes.

La música como tal, puede expresar emociones, afectos y estados de ánimo; con ella, los docentes pueden fomentar los valores en los niños vinculándolos con la música; al mismo tiempo, revelar sus competencias y orientar hacia el fortalecimiento de los elementos axiológicos desde su 
entorno. En función de ello, se intenta con este estudio proponer algunas alternativas para trabajar la música como herramienta al fortalecimiento de los valores en el nivel de educación básica.

Tomando en cuenta esta premisa, el Diseño Curricular del Sistema Educativo Bolivariano del (2007), está orientado en el proceso de refundación de la República como fin supremo del Estado y plantea la formación de un ciudadano y una ciudadana con principios, virtudes y valores de libertad, cooperación, solidaridad, convivencia, unidad e integración, que garanticen dignidad y bienestar individual y colectivo (pág. 11). Al respecto, en el trabajo del día a día, los niños y niñas son autores de su propio aprendizaje, con vigilancia a las particularidades individuales y el respeto como seres únicos dentro de un contexto familiar y comunitario; se presenta una constante colaboración hacia el desarrollo de todas y cada una de las actividades que se ejecutan a diario en el aula de clase.

Desde este punto de vista, dentro de las actividades pedagógicas que deben ser consideradas primordiales en el nivel de educación básica está inmersa la música, que además de ser un lenguaje entendido y bien recibido es una herramienta indispensable e invaluable en todos los aspectos del desarrollo integral, como son la creatividad, la socialización, el lenguaje, la memoria, los valores, entre otros, ya que está integrado por tres aprendizajes esenciales para el desarrollo del ser social: hacer, conocer y convivir, éstos tienen en la música un aliado fundamental que va unido al proceso de desarrollo y aprendizaje de niños y niñas.

Para justificar este precedente, es importante señalar que las principales expresiones del ser humano para conectarse con su contexto pasan por el llanto, el grito, la risa, el movimiento, palmear, y otras que son expresiones inherentes al género humano, vale aquí citar a Martorell (2009: 54), quien señala que:

En el contexto escolar, la presencia de actividades significativas que 
promuevan el proceso de enseñanza-aprendizaje depende de la acción que ejerce un docente mediador y que se complementa con el contexto sociocultural que rodea al educando. En la medida en la que se propicien experiencias de vida se dará lugar al despliegue de las potencialidades propias del niño de acuerdo con sus conocimientos previos y su nivel de desarrollo evolutivo. Al respecto, Gallego (2002: 98), dice:

...un buen docente es aquel que organiza los conocimientos y valores que los alumnos han ido adquiriendo a través de su historia personal en amplios esquemas que ayuden a analizar, comprender y transformar la realidad; presenta valores alternativos para vivir en sociedad... Es una persona que, con su intervención en el aula, su actitud y su orientación, provoca y facilita la reconstrucción del pensamiento, sentimientos, actitudes, valores y conductas.

Siendo así, muchas son las alternativas que tiene el docente a la hora de crear e implementar estrategias en función de lograr un mayor grado de respuestas en cuanto al aprendizaje se refieren. Una de ellas es la música, utilizada esta como recurso activo para estimular, llamar la atención, facilitar la participación, elevar el trabajo creativo, en fin, motivar permitiendo con ella salir del método tradicional de enseñanza e innovando con mecanismos que sean agradables y placenteros para los educandos, generando respuestas a las demandas que plantea el nuevo Sistema Educativo.

Una vía para lograr el proceso de aprendizaje sería entonces, la música como recurso, pues esta favorece la interacción grupal, enriquece la imaginación y permite el desarrollo de conductas abiertas hacia el aprendizaje. La música es una fuerza recóndita que ejerce una influencia poderosa en el individuo, ya desde el desarrollo del nuevo ser se puede evidenciar respuesta ante el estímulo de la música. Ella desempeña un papel importante en el sujeto e influye en su mente y en su pensar: por este fuerte efecto positivo, puede utilizarse la música como recurso metodológico en la aplicación de estrategias 
dentro y fuera del aula.

Señala Casas (2001: 79), que la música se encuentra presente en todas las actividades de la cultura del hombre como el juego, expresiones religiosas y expresiones emocionales, entre otras, mencionando que relacionarse con el mundo de la música es acercarse de manera más profunda al género humano; de manera que una buena orientación sobre el proceso de enseñanza y aprendizaje lo contribuye la música, pues conduce a mejorar la capacidad de concentración, autoestima, disciplina, respeto y socialización. El docente desempeña un papel muy importante en el proceso enseñanza -aprendizaje. Muchas veces el aprendizaje no llega igual al niño y la niña, pues cada uno aprende de acuerdo con su propio ritmo; de allí, la necesidad de aplicar otras técnicas y recursos que faciliten una mejor comprensión de los contenidos planteados.

Por ello, Guerrero (2007: 12), afirma que "la música, conjuntamente con la rítmica y las artes plásticas y dramáticas plantea un lugar esencial en la educación armoniosa de los niños y niñas" constituye no sólo un significativo factor de desarrollo, sino también un medio para calmar las tensiones, lograr equilibrio y controlar el exceso de energía en los niños; el mismo autor explica que la música es un medio que permite la formación integral del niño (a), pues no se enfocará únicamente en lo cognitivo sino también en lo afectivo y social.

\subsection{Propósito del estudio}

Dar alternativas de enseñanza aprendizaje utilizando la música urbana para el fortalecimiento de valores en el 3er en la educación básica.

\section{Marco teórico}

En este apartado se plasman los aportes teóricos que sustentan el estudio, así como las teorías que representan todos los soportes de autores quienes tratan el tema de la educación, así como de la música fuente de 
enseñanza/aprendizaje.

\subsection{Aproximación a la concepción de Música}

En su sentido más amplio, la música nace con el ser humano, y ya estaba presente, según algunos estudiosos, mucho antes de la extensión del ser humano por el planeta, Es por tanto una manifestación cultural universal. Para Comellas, J. (2006), "se ha demostrado la íntima relación entre la especie humana y la música, y mientras que algunas interpretaciones tradicionales vinculaban su surgimiento a actividades intelectuales vinculadas al concepto de lo sobrenatural" (pág. 121). Más adelante agrega el mismo autor que "para el hombre primitivo había dos señales que evidenciaban la separación entre vida y muerte: el movimiento y el sonido. Los ritos de vida y muerte se desarrollan en esta doble clave. En el llamado arte prehistórico danza y canto se funden como símbolos de la vida mientras que quietud y silencio se conforman como símbolos de la muerte." (pág. 127).

Puede decirse que el hombre primitivo, al igual que los habitantes autóctonos del país, encontraba música en la naturaleza y en su propia voz. También aprendió a valerse de rudimentarios objetos (huesos, cañas, troncos, conchas...) para producir sonidos. Hay constancia de que hace unos 50 siglos en sumeria ya contaban con instrumentos de percusión y cuerda (lira y arpa). En la prehistoria aparece la música en los rituales de caza o de guerra y en las fiestas donde, alrededor del fuego, se danzaba hasta el agotamiento. La música está basada principalmente en ritmos y movimientos que imitan a los animales. Las manifestaciones musicales del hombre consisten en la exteriorización de sus sentimientos a través del sonido emanado de su propia voz y con el fin de distinguirlo del habla que utiliza para comunicarse con otros seres. Los primeros instrumentos fueron objetos, utensilios o el mismo cuerpo del hombre que podían producir sonidos. 


\subsection{La Música dentro de la Educación}

La música es un arte que se transforma de generación en generación por todo el mundo, se adecua a la cultura de las civilizaciones y se presta como un medio de comunicación y expresión para el beneficio de las mismas. Ante este hecho la música se ofrece como una herramienta de enseñanza y aprendizaje que debe ser estudiada para su mejor rendimiento dentro de las aulas, razón por la cual se presenta a continuación el estudio de algunos autores e investigadores que tratan la temática de la enseñanza musical.

Es así como, en la educación, y de acuerdo con el Currículo Básico Nacional planteado por el Ministerio de Educación (1997), la música puede ser empleada como elemento material y mediador de ciertas cualidades articuladas de la sonoridad, timbre, tono, intensidad, duración y ritmo. La Música, plástica y artes escénicas constituyen ámbitos artísticos bien diferenciados que, también en la práctica educativa, deberían distinguirse. Sin embargo, en la educación básica quedan integradas en una sola área; lo importante es que se dé continuidad al análisis y a la reflexión sobre la producción artística.

Es necesario señalar, que la música, la plástica, y las artes escénicas no deben excluir la iniciación a otras expresiones artísticas como la danza, el ballet, la ópera, hasta la propuesta más avanzada de un espacio escénico cargado de elementos plásticos y sonoros considerando la arquitectura y el urbanismo. Para Matos (1998a: 187), la música como estrategia educativa debe comenzar a realizarse con los elementos básicos de la música, duración, intensidad, altura, timbre, ritmo; aplicados en unidades musicales simples: la interacción con estas unidades conduce al niño y niña a crear o a componer unidades más ricas y complejas, que a su vez pueden ser relacionados con los elementos cotidianos que los rodean; en la escuela, en el hogar o en su comunidad.

Por tanto, puede considerarse que la música, como recurso 
pedagógico, enriquece la formación integral del niño, no solo por su aspecto formativo sino también por su aporte en su sano desarrollo de la personalidad. Según un estudio citado por Matos (1998b) y realizado por Séller en 1990, entre las ventajas más significativas de la música está el desarrollo del aspecto intelectual, socio afectivo, psicomotor, de crecimiento personal y formación de hábitos; definitivamente es una herramienta que ofrece muchos recursos y aplicaciones para la formación en valores en la educación básica.

En el aspecto intelectual, la misma Séller considera que la música desarrolla una relación apropiada con el propio organismo (autoestima), permite la improvisación de respuestas creativas a situaciones imprevistas; favorece la atención, observación, concentración, memorización, experimentación, el conocimiento y percepción de conceptos globales como tiempo, espacio, y parciales como alto-bajo, suave-fuerte, claro-oscuro, gordoflaco, largo-corto, el lenguaje y desarrollo del cálculo, la agilidad mental y la creatividad, permite evaluar resultados mide capacidades, ayuda al conocimiento de sí mismo, enseña a pensar.

En la formación en valores, la música sirve como indicador de las capacidades creativas del estudiante y seguridad en sí mismo. Puesto que la misma cumple una función muy importante en el desarrollo socio-efectivo del niño al enseñar a diferenciar roles y definir responsabilidades, lo capacita para una mayor y mejor participación en el aula, en la relación con los compañeros y hasta con los mismos adultos al compartir o interactuar con ellos a través de juegos y actividades musicales (canto y ejecución instrumental), dirigidas fundamentalmente a ejercitar destrezas.

La música le produce al estudiante placer y satisfacción, despierta su observación y aceptación de todo cuanto lo rodea; le permite seguridad al desplazarse y ubicarse en el tiempo y en el espacio. Le facilita la integración grupal al compartir cantando y tocando los instrumentos con los compañeros, lo que refuerza, a su vez, la noción de trabajo cooperativo y otros indicadores 
de buena convivencia, como el respeto a la diversidad y el amor a sus semejantes. El estudiante se sensibiliza ante el arte y la naturaleza, descubre un nuevo medio de expresión y comunicación, fortalece su autoestima, conoce y expresa sus capacidades, demuestra su perseverancia en el alcance de metas; se motiva a superar dificultades cuando, al participar en producciones artísticas, se esfuerza en aplicar correctamente los elementos básicos de la música.

La música permite descargarse, relajarse, expresar sentimientos y canalizar sus energías de una manera apropiada, le sirve como medio para expresar el respeto hacia vida de los demás y la suya. La música está entre las opciones a las que puede acceder el estudiante ante actividades y consumo de sustancias que ponen en peligro su vida; desarrolla el sentido de la justicia, promueve la libertad, alivia el temor y la timidez, canaliza la agresión, desarrolla voluntad y autocontrol, enseña a delimitar libertades y a tomar decisiones, ofrece una visión del mundo y canaliza el logro de metas.

Es así la música permite representar situaciones personales para aprender de ellas, situaciones que se viven en el hogar, en la comunidad donde se desenvuelve el estudiante y por supuesto en el aula de clases; esta aplicación de la música de poner al estudiante en contacto con su realidad inmediata es propicia para la formación en valores, ya que se evidencia un sentido netamente práctico. Por tanto, los elementos básicos de la música pueden ser aprovechados en la formación en valores, ya que, por medio del sonido, el ritmo, la melodía y la armonía, todos ellos inseparables en la música, se enriquece e impulsa la vida interior del ser humano, y se estimula su voluntad, sensibilidad, amor, inteligencia y su imaginación creadora.

\subsection{Importancia de los Valores en la Educación Básica}

La adquisición de valores y cuales valores llegue a asumir la persona es un hecho de gran relevancia que no debe dejarse al azar. La educación en 
valores, ha de ser un proceso consciente y planificado que elimine cuanto sea posible, la intervención del currículum oculto de efectos no deseables. Es necesario, por lo tanto, que los especialistas en educación reciban la formación que les capacite para la enseñanza en los valores. En referencia, Aguilar (1996), señala que, en el análisis de grupos sociales, los valores son importantes porque son elementos movilizadores o desmovilizadores que inciden sobre el actuar condicionándolo.

De acuerdo con lo antes expresado, los valores y su significación están íntimamente relacionados pues su interrelación explica en gran medida, el desarrollo personal y como consecuencia el posible desarrollo social. Paz (1993a), señala que los valores son de gran importancia porque determinan la rectitud de los objetivos. Sin valores podemos generar una visión inmoral y poco ética. Estos son los que rigen nuestras conductas, permiten diferencias reales entre lo bueno y lo malo, entre lo próspero y lo decadente, entre lo social beneficioso y lo insuficiente o dañino.

En su opinión Marcano y Jiménez (2005: 10), indican que cuando los valores son adquiridos a través del desarrollo coherente y permanente de actitudes, éstos se reestructuran con la personalidad y se pueden transformar en capacidades propias. La persona se va capacitando para ser solidaria, responsable, autónoma, independiente. Para estos autores "los valores determinan el desarrollo de las capacidades, los comportamientos, las conductas y las actitudes". La importancia de los valores está inmersa socialmente, en el contexto familiar; esta radica en que la familia como grupo social constituye la confluencia de generaciones diversas, con valores diversos que consciente o inconscientemente, reproducen los valores de la sociedad y éstos a su vez, son construidos sobre aspectos deseables de la sociedad a fin de mantener un equilibrio en su estructura organizativa.

Es pertinente señalar en forma específica alguna de las características que describen a los valores, tomando en cuenta la relevancia del tema dentro 
de la investigación que se propone. Al respecto Villalpando (1996), dice que los valores son entendidos como entes ideales y que es necesario reconocer en ellos la posibilidad de su conocimiento. Además, éstos están dotados de una existencia en la mente del hombre y únicamente reconocida por ella. Puede ser motivo de un reconocimiento de validez general.

También señala el citado autor a los valores, las siguientes características generales: la posibilidad que tiene los valores de representar una cualidad positiva reconocida en una creación cultural, o contrariamente una cualidad negativa (polaridad); el grado de intensidad o de abundancia, con que se pueden dar los valores; cada valor es poseído o asentado, a título de cualidad en un bien cultural; los valores son objetos de una ordenación particular (jerarquización), tiene un carácter inmaterial.

\subsection{Factores que inciden en la formación de valores en la educación}

Aun cuando educar en valores, supone una reestructuración educativa sobre las pretensiones que se desean lograr con la formación de ellos muchas veces escapa de la educación formal el control de determinados factores presentes en el medio y que interfieren en el logro de los objetivos pautados por la escolaridad, para la formación de un ciudadano apto para la vida, con valores de autenticidad y superación.

Por su parte, Ramos (2005: 52), considera que el ambiente tiene un profundo efecto educativo, sobre todo en el aspecto de los valores. A través del ambiente, el individuo y el grupo se ven presionados en el sentido de la vivencia y aceptación de ciertos valores y el rechazo de otros. Considerando la necesidad de educar en valores como respuestas ante la influencia de valores negativos en la formación del ciudadano, la reforma educativa en el Nivel de Educación Básica, propone una educación integral que forme valores positivos mediante vivencias en la práctica.

Al respecto Herrera (1991: 13), expresa que la sociedad actual posee, 
como todas las sociedades y culturas, un sistema de valores. Considera que la educación escolarizada es la institución encargada de transmitir los valores sociales que se supone caracterizan la sociedad formal en la que se vive. Sin embargo, la sociedad moderna y urbana ha ido creando otras instancias que también se encargan de transmitir valores de manera explícita o encubierta, como la publicidad, la propaganda política o las ideologías socioeconómicas, la música, la cultura estética, industrial y urbana. Además, considera que otras instancias son más eficaces algunas veces en el logro de la transformación de valores que la propia institución educativa.

Respecto a ello es pertinente considerar ciertos valores de procedencia positiva, los cuales según Paz (1993b: 23), se clasifican en: microvalores como: solidaridad, participación, disciplina social, justicia, lealtad, creatividad, y macrovalores como: libertad, justicia social, paz, Unidad Nacional. Expresa además, que los microvalores y macrovalores señalados corresponden a los de mayor significancia, refiriendo que los primeros, representan lo cotidiano que mueve a cada persona en su lucha por ser feliz y los segundos, representan los lazos que rodean el bienestar de la comunidad.

En consonancia a lo señalado, y de acuerdo con las necesidades sociales, el Nuevo Diseño Curricular ha considerado en el documento que sustenta la nueva reforma educativa, los valores antes mencionados, los cuales fueron reseñados en las bases teóricas relativas a la educación en valores y que pretenden ser alcanzados por medio del desarrollo de los ejes transversales a través de la praxis pedagógica.

\section{Recorrido Metodológico}

La presente investigación fue de tipo descriptivo, definido por Sabino (2000: 65), como "La descripción de las características fundamentales del conjunto homogéneo del fenómeno utilizando criterios sistemáticos que permitan poner de manifiesto su estructura o comportamiento". Así entonces, 
en este trabajo el autor describió propiedades o características del grupo estudiado. En cuanto al nivel de la investigación, el autor citado indica que esto se refiere al grado de profundidad con que se aborda un objeto o fenómeno, indicando si se trata de una investigación exploratoria, descriptiva o explicativa. La población, según Hurtado (1997: 140), "es una porción de la población que se toma para el estudio, la cual se considera representativa. Tamayo y Tamayo (2006: 92), define la población en términos estadísticos como: "La totalidad del fenómeno a estudiar en donde las unidades de población poseen unas características comunes, la cual se estudia y da origen a los datos de investigación". En este estudio, la población está representada por los docentes de la Escuela Bolivariana "Vivienda Rural".

Por otro lado, la muestra la conforman seis docentes que imparten clases en tercer grado secciones A, B y C. Al respecto Hernández, Fernández y Baptista, (2010: 212), definen la muestra como "un subgrupo de la población, que permite generalizar sobre ésta, los resultados de una investigación". En este caso se toma tercer grado por considerar que es un curso en el cual se debe fortalecer el proceso de aprendizaje ya que vienen de grados anteriores en los cuales han utilizado el juego y la música para desarrollar habilidades y destrezas $\left(1^{\circ}\right.$ y $2^{\circ}$ grado $)$ y continuarán a grados superiores $\left(4^{\circ}, 5^{\circ}\right.$ y $\left.6^{\circ}\right)$ donde desarrollarán lo aprendido en 3er grado, considerándolo así un grado base para la madurez del aprendizaje del niño y la niña.

\section{Resultado}

Se pudo observar que los docentes sí aplican estrategias lúdicas; sin embargo, en las estrategias a través de la música, no son muy empleadas, además los maestros encuestados plantean que sí es importante desarrollar la educación musical en la institución. En estos resultados se evidencia el poco uso de indicadores para el desarrollo social y afectivo de los estudiantes, las actividades artísticas y creativas para el fomento de la expresión musical y la 
integración del niño en su salón de clase por parte de sus educadores; sin embargo, los docentes poseen conciencia de la importancia de las estrategias musicales. Asimismo, los encuestados manifiestan que usan diversas estrategias como los cuentos, Role play, dramatizaciones trabajo colaborativo como parte de su cotidianidad para reforzar los valores en los niños, al igual refuerzan los valores amistad, solidaridad, honestidad, bondad en el aula de clase, lo cual muestra la importancia que les dan a los elementos axiológicos como parte de la formación de los pequeños.

\section{Conclusiones}

Con referencia al primer objetivo específico, el cual se destinó a diagnosticar el uso de la música como herramienta de enseñanza en el 3er grado se pudo confirmar que son pocas las oportunidades en que se toma en cuenta el uso de la música como herramienta para la enseñanza dentro del aula de clase en la nombrada escuela. En relación a ello, la institución no se le saca provecho al uso de la música que puede ser utilizada con mayor frecuencia en los niños del 3er grado para fortalecer la enseñanza, obviando así, la importancia que esta tiene para un aprendizaje significativo; convirtiéndose las aulas de clase -la mayoría de las veces- en rutinarias para los niños, ya que no se les brinda la oportunidad de aprender de una manera divertida desde el desarrollo de la inteligencia auditiva y la expresión vocal mediante actividades educativas y al mismo tiempo de suficiente agrado tanto para los docentes como los estudiantes.

De acuerdo con el segundo objetivo de esta investigación, el cual buscó determinar las estrategias de enseñanza de valores en el 3er grado; se reflejó que en dicha institución sí se realizan actividades para la enseñanza y fortalecimiento de valores, lo cual resulta bastante favorable para el desarrollo y convivencia, teniendo en cuenta que también se involucran a los padres y representantes de los niños y niñas. Los docentes realizan una labor 
importante ya que, el interés por organizar eventos que incluya a los padres a su vez que a los niños en actividades tales como lecturas, dramatizaciones, narraciones, reflexiones para fortalecer los valores es una acción que promete de manera positiva y permite afianzar las relaciones entre estudiantes-padres y representantes; estudiantes-docentes; docentes-padres y representantes logrando un clima bastante agradable donde se reflejen los valores y fortaleciendo así la triada escuela-familia-comunidad.

En cuanto al propósito de la realización de una propuesta se realizó la construcción de textos en verso o prosa para su transformación en canción, se trabaja con los niños implementando el trabajo colaborativo, el cual fortalecerá vínculos de convivencia, así como las relaciones socio afectivas; los niños crearán un texto que más tarde convertirán en un rap, Hip Hop, entre otras composiciones musicales. En este apartado se presentará una canción que fue creada por niños del 3er grado en compañía del investigador; en la misma, se reflejan una serie de valores.

Canción: Amor respeto y convivencia:

Autores: Estudiantes del 3er grado de la Escuela Bolivariana "Vivienda Rural".

CORO:

Hoy da inicio; ese gran día Voy a despertar: como esperanza

Hoy decido, amar a mis padres Cambiar la historia

Siendo la persona

De bien valorar, ajah ajah De bien valorar

\section{ESTROFA:}

Oh esto me ha enseñado, que sea valorado Mi amigo como hermano, la profe lo ha evaluado

De escuela soy soldado, y así seré alistado Ahora escucha escúchame bien 
¿El amor es hermoso? Cuando respeto a mi amigo ¿Yo quiero a la vida? Cuando respeto a mis padres ¿Se llega a la cima? Cuando convivo a lo grande.

Es importante señalar que, la construcción presentada es un ejercicio que se diseñó durante la investigación; como puede observarse, en la misma se evidencian elementos axiológicos como el respeto, la convivencia y el amor como parte de los valores inmersos dentro del texto que se convertirá en música. En este sentido, es importante que el docente como mediador del aprendizaje emplee con frecuencia la música, aportando un ambiente positivo y relajado en el ámbito educativo, a la vez que favorecen la integración sensorial necesaria y promueve las diferentes interacciones.

\section{Referencias}

Aguilar S. (1996). Los Valores en el Medio Familiar. Revista de Ciencias Sociales. Caracas. Volumen IV. Número 5. [En Línea]. Recuperado de: http://www.ts.ucr.ac.cr/binarios/docente/pd-000159.pdf

Casas, M. (2001). La Música en la escuela. Barcelona: Graó.

Comellas J. (2006). Nociones sobre la Música. México: Paidos.

Currículo Nacional Bolivariano (2007). Diseño Curricular del Sistema Educativo Bolivariano. Educere, 11(39), 751-775. Recuperado de: http://www.scielo.org.ve/scielo.php?script=sci arttext\&pid=S131649102007000400020\&lng=es\&t|ng =e

Ministerio de Educación (1997). Currículo Básico Nacional. Venezuela. [En Línea]. Recuperado de:

http://salonvirtual.upel.edu.ve/mod/book/print.php?id=4263

Gallego, C. (2002). Familias y docentes en una tarea común: la educación musical del niño y la niña. Revista mensual de publicación en Internet Número 26‥ Recuperado de: 


\section{http://www.filomusica.com/filo26/cristi.html}

Guerrero L. (2007). La música un abordaje en la escuela de hoy. Esperjo.

Colombia.

Hernández, R., Fernández, C. \& Baptista, P. (2010). Metodología de la

Investigación. Ciudad de México, México: Editorial McGraw-Hill.

Herrera, M. (1991). La Cultura escolar: Práctica y valores educativos.

Conferencia presentada en las Jornadas Nacionales Perspectivas para

Mejorar la Calidad de la Educación Venezolana, Venezuela:

Universidad del Zulia.

Hurtado de B., J. (2008). Metodología de la Investigación Holística.

Venezuela: Editorial Sypal.

Marcano, S. \& Jiménez, M. (2005). La valija didáctica para la formación en educación ciudadana y rescate de valores (manual). Venezuela: Apoyo Gráfico Integral. C.A.

Matos, R. (1998a,b). Juegos Musicales como recurso pedagógico en el preescolar. ISBN: 980-273-454-3, págs. 148. Caracas: Fedupel.

Martorell, S. (2009). Música del mundo para niños. Zaragoza: Publicaciones universitarias de Zaragoza.

Paz, S. (1997a,b). Los Valores Fundamentales. Revista Universidad. Volumen II. Venezuela: Ediciones EAFIL.

Ramos L. (2005). Cómo enseñar y transmitir valores. Guía para padres y maestros. México: Editorial Trillas.

Sabino, C. (2000). El Proceso de la Investigación. Venezuela: Editorial Panapo.

Tamayo T., \& Tamayo M. (2006). Metodología de la Investigación Científica. Grupo Noriega Editores. México: Editorial Limusa S.A. de C.V.

Villalpando J. (1996). El Mundo de los Valores. Revista Mexicana de Pedagogía. México: Iresie. Vol.: 7. Núm.: 28, págs. 14-20. 


\section{Carol del Carmen Terán González}

e-mail: carolteranula@gmail.com

Nacida en la ciudad de Valera Edo Trujillo, Venezuela.

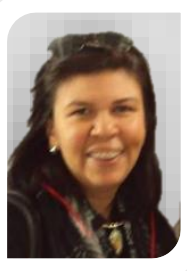

Cursó estudios de Doctorado en Educación en la

Universidad Rafael María Baralt, posee Maestría en

Docencia para la Educación Superior en la misma universidad, Maestría en Literatura Latinoamericana en la Universidad de los Andes y Licenciatura en Educación Mención Castellano y Literatura ULA-NURR investigadora del Centro de investigaciones literarias y lingüísticas Mario Briceño Iragorry. (CILL) ULANURR, Jefe de la unidad de investigación y proyecto de la Casa de Historia Trujillo, articulista e investigadora con 14 años de servicio en el área de educación y literatura. Ponente en eventos nacionales e internacionales. Docente invitada en pregrado y Postgrado, ULA, UNERMB, UNESR. Coordinadora de la Línea de investigación Memoria, Educación y Discursos emergentes (UNERMB), miembro de la Línea de investigaciones Ciudadanía, hermenéutica y proyectos sociales. (UNERS). Ha realizado artículos en distintas revistas arbitradas del país. 


\section{Josefa María Ramírez Becerra}

e-mail: josefa chepa@hotmail.com

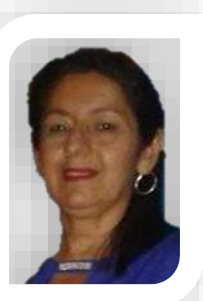

Nacida en Valera, estado Trujillo, Venezuela. Magíster en

Docencia para la Educación Superior Licenciada en

Administración de empresa UVM. Especialista en

Gerencia de Empresas UVM. Doctorante en Ciencias de la Educación. Docente contratada Tiempo completo del Instituto Universitario de Tecnología del Estado Trujillo Extensión Trujillo. 


\section{Lom Hill Parra}

e-mail: lom 203@hotmail.com

Nació en Trujillo, estado Trujillo, Venezuela. Licenciado en Educación Integral ULA-NURR. Vigilante en la Universidad de los Andes, Docente de educación Básica. Perteneciente a la línea de investigación Memoria, educación emergente.

\footnotetext{
El contenido de este manuscrito se difunde bajo una Licencia de Creative Commons ReconocimientoNoComercial-Compartirlgual 4.0 Internacional
} 